\title{
Register der Personennamen und geographischen Begriffe
}

Aalen 132

Aamodt, Sandra 691

Abbt, Thomas 80

Abertham (Abertamy) 23-26, 28

Aberystwyth 492

Abessinien 196, 302, 401 f., 546

Ackerknecht, Erwin 292

Acton, John Emerich Edward, Lord 172

Adelbert von Chamisso 685

Adenauer, Konrad 308, 320, 394, 467, $5^{19}$

Adiga, Aravind 687

Adorno, Theodor W. 529

Ägina 226

Ägypten 213, 227, 234, 351, 354, 359-361, $457,463,586,681,699$

Äthiopien 402

Ahus, Adele 585

Aischylos 702

Akutagawa Ryunosuke $5^{87}$

Albers, August 289-295, 297-299, 301, 303f., 313, 315, 329, 331, 336, 341, 344, 348f., $353,358,396,541,725$

Albertus Magnus 692

Albrecht, Barbara 582

Albrecht, Günther 582

Alewyn, Richard $558 \mathrm{f}$.

Alexander der Große, König der Makedonen

205, 333, 495, 499, 513, 552, 554, 703

Alexander II., Zar 175

Alföldy, Géza 513

Alt, Albrecht 234, 351 f., 64,2

Alt, Jörg 665

Alt, Johannes $25^{1}$

Alt, Peter-André 709

Althaus, Hans-Peter 697

Altheim 53

Altheim, Franz 492

Altmann, Bruno $338 \mathrm{f}$.

Amsterdam 681

Anders, Günther 320, 530-532, 538, 546, $711,73^{2}$

Angermaier, Heinz 526

Angola 317

Anna Amalia von Braunschweig-Wolfenbüttel 245,679

Ansbach 137
Apelt, Willibalt 307, 435

Appiah, Kwame Anthony 663, 694, 736

Aragon, Louis 477

Arasse, Daniel $5 \mathbf{2 8}$

Archimedes von Syrakus 690

Arendt, Hannah 530 f.

Aristophanes 217,702

Aristoteles 499, 692, 702

Arndt, Adolf 538

Arnold, Heinz-Ludwig 581

Arnold, Melvin 320

Arras $\mathbf{3 2 6}$

Artois $\mathbf{3 2 6}$

Assmann, Aleida 705, 708, 711

Assmann, Jan 644, 698f., 705, 708, 710, 736

Atabay, Cyrus 589

Athen 211, 213, 216, 226, 229, 539, 555, 630, $638,648,702$

Atkins, Stuart 562

Augsburg 46, 51, 55, 60, 63, 66-71, 77, 83, 94f., 112, 119, 122, 132 f., 137, 158, 421, $526,680,706$

Augustin, Ernst 670, 686

Augustus, röm. Kaiser 205, 231, 508, 513, 634,683

Auschwitz 552, $647 \mathrm{f}$.

Austin, Michel $63^{2}$

Australien $63^{2}$

Autenrieth, Georg $211 \mathrm{f}$.

Avenarius, Ferdinand 274

Baberowski, Jörg 711, 714

Bach, Johann Sebastian 293, 314, 323

Bachmann, Ingeborg 46o, 687

Bachofen, Johann Jakob 292 f., 296, 334-336, 341

Bade, Klaus J. $712 \mathrm{f}$.

Baden 64, 158, 165,178

Baden-Baden 452, 497, 668

Badian, Ernst 555

Baer, Joseph $3^{6} 5$

Bärend, Ernst 493

Baethgen, Friedrich 546

Bätschmann, Oskar 682

Baeumler, Alfred 334-336

Bahners, Patrick 696

Bahr, Egon 584 
Bahrdt, Hans Paul 562

Bajohr, Frank 373

Baldingen 63f., 94, 144, 255

Balzac, Honoré de 446

Bamberg 172,684

Barcelona 711

Barmen 468

Barner, Wilfried 525,696

Bartel, Christof 23

Barth, Marquard 170

Barthélemy, Guy 320

Baschwitz, Kurt 384

Basel 114, 216, 218, 705

Batt, Kurt 584

Baudelaire, Charles 295

Bauer, Adolf 211, 213, 230

Bauer, Friedrich 134,149

Baumbach, Adolf $366,369,376$

Baumeister, August 207, 209, 236

Baur, Eva Gesine 716

Baur, Friedrich Christian 160

Baur, Wilhelm 437

Bayern 45f., 81 f., 89, 98, 114-117, 119, 131, $133,136-139,144-146,151,153,156 \mathrm{f}$., $167,169-178,184,186,190,196,201$, 214 f., 255,257 f., 26o, 272, 298, 306, 309 f., 326 f., 331, 334, 337, 340, 342, 351 f., 386, 396, 398, 405f., 416, 419, 4,23, 4,27 f., 431, 434f., 436 f., 457 f., 475, 518, 520 , $5^{25}-5^{28}, 540,543,548,569,609,676$, $689,706,718,7^{24}, 7^{2} 7-729,73^{1}$

Bayley, John 686

Bayreuth 113f., 122, 187, 303

Bechert, Karl 320

Beck, Alexander 671

Beck, Anja 671

Beck, Anna 23

Beck, Babette Lucie Eugenie, geb. Heinzelmann 133, 139, 159, 170, 325

Beck, Carl 133, 187, $255 \mathrm{f}$.

Beck, Carl Gotthelf $3_{1} \mathrm{f},{ }_{35}$ f.

Beck, Carl Gottlob 18-2o, 23, 26, 31 f., 35-40, 42-88, 91-103, 105-112, 114f., 118, 120, 122, $271,724,726 \mathrm{f}$.

Beck, Carl Heinrich 113, 115-123, 127-129, 131, 145

Beck, Carl Heinrich August 20, 127 f., 133-139, 141-144, 148, 150 f., 153, 157-159, 201, 206, 271, 297, 724

Beck, Christian Friedrich (Sohn von Johann Gottfried Beck) $3^{1}$

Beck, Christian Friedrich (Sohn von Carl Gottlob Beck) 113

Beck, Christian Gottlob 114

Beck, Christoph Friedrich 114

Beck, Clara 271, 294.
Beck, Erna 271

Beck, Esther Rosina, geb. Voigtländer 23, $28 \mathrm{f}$., $3^{1}$

Beck, Eugen 256

Beck, Eva, geb. Müller 34,2, 4,16 f., 4,22, 433

Beck, Eva 671

Beck, Friedrich 128

Beck, Georg August 114

Beck, Hans Dieter 342, 352, 382, 542, 547, $549,563,570-574,670-672,675,684$, 724,733

Beck, Hans-Georg 517, 546, 698

Beck, Hedwig, geb. Burger 271, 293

Beck, Heinrich 19f., 23, 197, 234-236, 271 f., 282, 287, 289-291, 293-295, 297-308, 311, 313f., 316-337, 340, 342-345, 348-354, $358-362,365,369-376,379-383$, $390 \mathrm{f}$., 395 f., 398, 401-4.05, 4.09-4.17, 4.20-4.22, 424-441, 445f., 448, 450-453, 455-458, 468 f., 472, 478 f., 481-483, 486-49o, 496, 500-502, 505-508, 515-527, 529-533, 537 f., $540-55^{2}, 557-560,562,564$ f., 567-575, 657,669,674-676,68o, 724, 729-733

Beck, Heinrich Gotthelf 114

Beck, Heinrich Theodor 114

Beck, Helga 67 I

Beck, Johann Daniel 113

Beck, Johann Georg 26-3o, 32

Beck, Johann Georg Wilhelm 127, 159, 187, 255, 264

Beck, Johann Gottfried 28f., $3^{1}$

Beck, Johanna Louise, geb. Heidenreich 35 f., 112,114 f., $117,121 \mathrm{f}$.

Beck, Johanna Maria Rosina 114

Beck, Jonathan $67 \mathrm{l}$ f.

Beck, Josephine Johanne, geb. Schertel 113

Beck, Juliane $67 \mathrm{l}$

Beck, Juliane Eberhardine 114

Beck, Julius $255 \mathrm{f}$.

Beck, Karoline Friederike 114

Beck, Katharina Barbara 36

Beck, Katharina Magdalena, geb. Heinzelmann 121 f., 127-129, 131, 133f., 139, 148, 725

Beck, Laura 271

Beck, Liselotte 34,2, 352, $570 \mathrm{f}$.

Beck, Mahrokh 671,685

Beck, Maria Magdalena, geb. Förster $27 \mathrm{f}$,, 30

Beck, Mathes 23

Beck, Nigin 671

Beck, Oskar 19f., 128, 133, 159, 167, 169, $173,187,191,201,205-210,214,216-220$, 222-224, 226, 233 f., 236, 243 f., 247-253, 255-273, 278 f., 281 f., 290 f., 293 f., 296 f., 
$325,329,33^{1}$ f., 334, 343, 412, 425 f., 570, 724,728

Beck, Susanne 342, 519, 540, 570 f., 573

Beck, Valentin 671

Beck, Veronika (Tochter von Heinrich Beck) $342,565,570$

Beck, Veronika (Tochter von Hans Dieter Beck) 671

Beck, Wolfgang 273, 310, 324, 342, 471, 510,

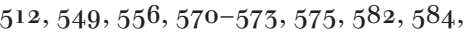
589, 597, 6о1, 6о5-607, 611, 616, 623, 625, 631, 641, 645f., 652, 654, 657-66o, 662, $66_{5}, 668,670-673,675$ f., 679 f., $683-685$, $687,694,701$ f., 712,715 f., $724,733-736$

Beckmann, Max 476

Beethoven, Ludwig van 716

Behn, Walter Richard 305

Beinhofer, Claus 666

Beisel, Dieter 690

Belgrad 234

Bell, Harold Idris 492, 495

Bellini, Giovanni 682

Beloch, Karl Julius 219

Belting, Hans 661, 681 f., 736

Ben-Sasson, Haim Hillel 643, 705

Benevolo, Leonardo $712 \mathrm{f}$.

Bengtson, Hermann 458, 485-495, 497-514, 518, 524, 546, 548, 551, 553 f., 595 . $629-633,635,637,639,698$

Benn, Gottfried 287, 457

Benz, Wolfgang 644,707

Benzmann, Hans 276

Berger, Friedemann 579, 581-583, $585 \mathrm{f}$.

Berger, Karl 247

Bergkau, Johann 28

Bergman, Ingrid $518 \mathrm{f}$.

Bergmann, Gunter 587

Bergson, Henri $\mathbf{3 2}^{2} 8 \mathrm{f}$., $5^{29} \mathrm{f}$.

Berlin 19, 37 f., 44, 80, 84f., 89, 98, 106, 108, $113,142,145,153,165 \mathrm{f} ., 182,186,189$, $193,196,211-213,219,226,235,243 \mathrm{f}$., $248,255,257,259,268,295,297,321,323$, $325,332,340$ f., 348, 350, 354-356, $365-367,369-372,376,378-380,382$, 387, 389, 391, 394f., 400, 405, 414, 419, $422,429,43^{2}, 437$ f., 448, 451, 462, 468, $470-473,477$ f., 492, 496, 517, $5^{22}, 5^{24}$, $542,556,560,582,587,592,622,642$, 644, 649, 681-684, 686, 696, 702, $707 \mathrm{f}$, $710,714,730$

Bern 311, 315, 502, 524, 561, 563, 682

Berner, Konstanze 665

Berve, Helmut 332, 335 f., 431, 433, 488-490, 492 f., 495 f., 503, 506, 544, 551-556, 633 , 636,639

Berve-Glauning, Anna Elisabeth $554 \mathrm{f}$.
Beth, Karin 680

Bethe, Erich 230

Bethel 141

Bethge, Hans 274

Bethmann Hollweg, Theobald von 289

Beutelsbach 70

Beutelspacher, Albrecht 690

Beyschlag, Daniel Eberhardt 45-47

Bezold, Raimund 664, 698

Bielefeld 592 f., 6o9, 613,617, 623, 626, 690

Bielenberg, Christabel 469

Bielenberg, Peter 469

Bieler, Manfred 471-476

Bieler, Marcella 473, 475

Bielschowsky, Albert 243-247, 250 f., 264, 403

Bierbaum, Otto Julius 377

Bieritz, Karl-Heinrich 587

Biese, Alfred 250-252, 264, $33^{1}$

Bikelas, Demetrios 208

Billerbeck, Paul 235, 334, 642

Birley, Anthony 555

Birner, Friedrich 114

Birner, Wilhelmine Rosine, geb. Beck 114

Birt, Theodor 228

Bismarck, Klaus von 274

Bismarck, Otto von 151, 164-166, 171-173, $175,180,183,194,246,274,338,385,408$, $604,614,709$

Bissing, Friedrich Wilhelm von 4,20

Bissingen 134

Bitburg 64.4

Bittel, Kurt 528

Bitterli, Urs 609

Bläsing, Theodor $15^{\circ}$

Blaschke, Olaf 19

Blass, Friedrich 211 f., 228

Blaubeuren 177

Bleckmann, Bruno 703

Bleutge, Nico 685

Blickle, Peter 591

Bloch, Marc 630, 705

Blochmann, Elisabeth 336

Blümner, Hugo $23^{0}$

Blunck, Hans Friedrich 407

Bluntschli, Johann Caspar 20, 153-167, $172-174,177,179-181,184,189-193,727 \mathrm{f}$.

Bobzin, Hartmut 589, 714

Bochum 693

Bock, Gisela $712 \mathrm{f}$.

Bode, Rudolf 401

Bodelschwingh, Friedrich von 14,

Böckh, August 218

Boeckh, Christian Gottfried $5 \mathbf{l}$ f.

Böhmen 23-26, zof.

Böll, Heinrich 477 
Bönig, Winfried 717

Böning, Holger 85

Böök, Fredrik 339

Bohrer, Karl Heinz 602

Bojer, Johan 337 f., 384, 40o, 684

Boll, Franz 223

Bollmann, Stefan 664, 69o

Bonhöffer, Adolf 230

Bonhoeffer, Dietrich 697 f., 709

Bonn 153, 211 f., 219, 251, 386, 400, 462, 471, $519,531,55^{1,622}, 662$

Boockmann, Hartmut 562, 594, 705

Bopfingen 113

Borasio, Gian Domenico $691 \mathrm{f}$.

Bordeaux 315

Borries, Erika von 709

Borst, Arno 562

Bosl, Karl 603

Boudot-Lamotte, Madeleine 4,13, 451

Bourdieu, Pierre 14, 16

Boveri, Margret $464 \mathrm{f}$.

Boyle, Nicholas 709

Bracher, Karl Dietrich 598

Bräker, Ulrich 697

Braig, Carl 34,1

Branca, Alexander von 669

Brandenburg 605

Brandenburg, Hans 247

Brandstetter, Oscar 290

Brandt, Hartwin 702

Brandt, Samuel 223

Brandt, Willy 478

Brasillach, Robert 400 f., 476

Brater, Karl 137-139, 153, 155, 157 f., 164, 170 f., 176 f., 181, 189, 208, 727

Brater, Pauline 208

Braumüller, Wilhelm von $289 \mathrm{f}$.

Brecht, Bertolt 457

Brednich, Rolf-Wilhelm 698

Breitenbach (Potůčky) $28 \mathrm{f}$.

Breitenbrunn 26

Breitkopf, Bernhard Christoph 38

Bremen 44, 243, 556, 573

Brenner, André 665

Brenner, Michael 649,651 f., 655

Breslau 38, 148, 243, 296, 385, 530, 551

Breslauer, Martin 367

Bresslau, Harry 317

Breysig, Kurt $297 \mathrm{f}$.

Brieg 244

Bringmann, Klaus 703, 715

Brissel, Max 191

Brodersen, Kai 716

Brönner, Heinrich Ludwig 109

Bronisch, Thomas 716

Broszat, Martin 464.
Brown, Peter 713

Browne, Thomas 54

Bruckmann, Alexander 159, 177

Bruckmann, Elsa 343, 4,17

Bruckmann, Hugo 343, 417

Brück, Michael von $695 \mathrm{f}$.

Brück, Regina von 696

Brugmann, Karl 211 f., 229

Bucerius, Gerd 694

Buchenau, Arthur 209

Buchner, Edmund $55^{2}$

Bücheler, Franz 214

Büchner, Georg 686, 709

Büchner, W.M. 14,8

Büchting, Hans-Ulrich 480, 524, 540, 547, $570,573,73^{\circ}$

Bülow, Arthur 54,8

Bürckstümmer, Christian 276

Büschgen, Hans E. 654

Bulle, Heinrich 231

Bullock, Alan 598

Bultmann, Rudolf 34,1

Burck, Erich 495f., 535 f., 550 f., 603

Burckhardt, Jacob 216, 218, 336, 705

Burger, Christian Heinrich Karl 113

Burger, Christiane Friederike, geb. Beck 113 f., 271

Burger, Hedwig s. Beck, Hedwig

Burger, Karl August 113 f., 271

Burke, Peter 14, 713

Burkert, Walter 696, 705, 711

Bursian, Konrad 209

Busch, Werner 682

Busch, Wilhelm 54,2

Buschor, Ernst 398, 423, 546

Busolt, Georg 211, 213, 230, 232

Butor, Michel 477, 541, 547

Caesar, Gaius Iulius 219, 553, 634, 637, 703

Caeyers, Jan 716

Caligula, röm. Kaiser 703

Calvin, Johannes 25, 32

Calw 14,8

Cambridge 510,555,630,632, 634

Cameron, Alan 510

Cameron, Averil 510

Canfora, Luciano 703, $713 \mathrm{f}$.

Capelle, Wilhelm 492

Caravaggio, Michelangelo Merisi da 682

Cardini, Franco 712

Carl Eugen von Württemberg 81

Carson, Rachel Louise 478,689

Cary, Joyce 4,00

Casanova, Giacomo (Chevalier de Seingalt) 582

Chaldäa 229

Chamberlain, Houston Stewart 253, 347, 354 
Champagne 326

Charleville 291

Chartier, Roger 14

Chastagnol, André 510

Chemnitz 108

Chile $3^{17}$

China 162 f., 322, 586

Chodowiecki, Daniel 367

Chopin, Frédéric 716

Christ, Karl 510, 629,633-639, 698, 703, 705

Christ, Wilhelm von 209-211, 214, 220, 225, $231 \mathrm{f}$., 236, $266 \mathrm{f}$.

Christensen, Arthur 234

Christian VII., König von Dänemark 60

Chruschtschow, Nikita Sergejewitsch 519

Cincinnati $646,65^{\circ}$

Classen, Walter 260

Clauss, Manfred $637 \mathrm{f}$.

Claussen, Johann Hinrich 717

Cohn, Leopold 229

Collier, Paul 711

Conrad, Michael Georg 274

Constable, John 682

Constant, Benjamin 156

Conze, Werner 591, 617

Cordes, Johann Gottlieb 276

Cory, Daniel 547

Craemer-Ruegenberg, Ingrid 692

Cragg, Tony 680

Craig, Gordon A. 591, 596-6o7, 609,611, 613f., 616-619, 694, 705, 733

Crome, Friedrich Leonhard 296

Crusius, Siegfried Leberecht 4,8

Cummings, Edward Estlin 688

Curtius, Ernst 216

Dach, Simon 560

Dachau $55^{2}$

Dänemark 6o, 139, 170, 579

Dahlmann, Friedrich Christoph 157, 205

Dahrendorf, Ralf 694

Dalmatien 292

Danzig 4,13

Darmstadt 6o, 631

Darwin, Charles 177 f., 478, $5^{21}$

Datterer, Franz Paul 270

David, Alexander 105

Davies, Norman 606

De Bono, Emilio 402

De Boor, Helmut 524f., 556-56o, 603

De Gaulle, Charles 322, 519

De Martino, Francesco 638

Deakin, Frederick William Dampier 598

Dechamps, Bruno 534

Defoe, Daniel 579, 585

Degas, Edgar 682

Dehler, Johann Ernst 35
Dehmel, Richard 274

Deidesheim 170

Delbrück, Ernst 194

Delbrück, Hans 194

Demandt, Alexander 512, 638,661, 698, 703, 707

Demandt, Barbara 661

Demosthenes 499, 702

Dempf, Alois $\mathbf{3 0 8}$

Denzer, Horst 569

Dessau 473

Deutsche Demokratische Republik (DDR) 321, 323, 471-476, 489, 579-589, 620f., $635,691,709$

Dhünens, Felix (Franz Sondinger) 405

Dickens, Charles 688, 710

Diederichs, Eugen 249, 258, 325, 337, 344

Diehm, Heinrika Sophia Katharina, geb. Beck 113

Diehm, Johann Friedrich 113

Diels, Hermann 214

Dießen 4,23

Diesterweg, Moritz 365

Dietzenbach 273

Diez 100

Dihle, Albrecht 535

Diller, Hans 495, 535

Dillingen 132, 186

Dilthey, Wilhelm 236

Diner, Dan 653, 655

Dionysios I. von Syrakus 499

Dippel, Horst $715 \mathrm{f}$.

Dischingen 54

Distel, Barbara 644

Diwald, Hellmut 597

Dixelius, Hildur 384

Dodds, Eric Robertson 499

Doderer, Heimito von $398 \mathrm{f} ., 4,22,445-460$, $476,5^{20}, 546-548,557,684,73^{2}$

Doderer, Maria Emma von, geb. Thoma $457 \mathrm{f}$.

Döblin, Alfred 581

Dölger, Franz 496

Döllinger, Ignaz von 172 f., 261 f., 270

Dönhoff, Marion Gräfin 469

Dohm, Christian Wilhelm 104

Dolp, Anton Jakob 69-71, 92 f., 96, 107

Dolp, Daniel Eberhard 70, 111

Dolp, Georg Friedrich 82

Dolp, Rosina Elisabeth 111

Donauwörth 55, 132 f., 174

Doppelmayr, Friedrich Wilhelm 119, 122

Dorn, Dieter 710

Dorpat 26o, 293

Dostojewski, Fjodor Michailowitsch 293, 446,476

Dove, Alfred 262 
Drawert, Kurt 685

Dreher, Eduard 396, 547

Dreher, Martin 703

Dresden 223, 335, 340

Drexel, Johann Karl $3^{6}$

Drexler, Hans 55of., 553

Droysen, Johann Gustav 193, 205, 709

Dubček, Alexander 475

Dublin 614

Dubus III, Andre 686

Duchesne, Louis 263

Duden, Konrad 149

Dülmen, Richard van 609

Dümmler, Ferdinand 226

Dürer, Albrecht $5^{27}$

Düring, Günter 396

Dürrenmatt, Friedrich 662

Düsseldorf 237, 491, 680

Dulles, John Foster 662

Dumas, Alexandre 459

Duras, Marguerite 477

Duttenhofer, Friedrich M. 177

Dwinger, Edwin Erich ${ }_{467} 67$

Dzierzon, Johann 135

Ebel, Hans Paul 19f., 370-372, 374, 376,382 , 4,14, 54,2 f.

Ebenhausen 668

Eberhardt, Siegfried 337

Ebert-Schifferer, Sybille 682

Eck, Werner $5^{13}$

Eckebrecht, Franz Josef 80

Eckstein, Richard 396

Eco, Umberto 593, $712 \mathrm{f}$.

Ecuador 377, $379 \mathrm{f}$.

Edzard, Dietz Otto 705

Eggert-Windegg, Walther 269, 273-275, 278-282, 304, 329, 344, 348, 353, 358, 541,725

Ehard, Hans $5_{18}$

Ehinger, Jacob Christoph 35

Ehmann, Hermann 697

Ehmcke, Fritz Helmuth 294, 403, 431, 438

Ehrenberg, Victor 554

Ehrhardt, Albert 221, 232

Ehrmann, Marianne $55 \mathrm{f}$.

Eichendorff, Joseph von 247, 709

Eichmann, Adolf 531

Eichmann, Klaus $53^{1}$

Eichstätt 134, 718

Einstein, Albert 690

Eisenach 165, 281, 283

Eisenreich, Herbert 546

Eisner, Kurt 288, 290

Ellwangen 55, 132

Elsass 236, 311, 315, 317, 406, $43^{6}$

Elze, Walter 401
End, Gustav 4,28-432, 441, 454 f., 457, 461, $466-468,470-47^{2}, 47^{6}, 47^{8}-482,515,531$, $534,541,545,548$ f., 560, 569-571, 725, $731 \mathrm{f}$.

Ende, Werner 589, 714

Engel, Eduard 252, $406 \mathrm{f}$.

England 52, 128, 276, 315, 408, 494, 529, $55^{2}, 554$ f., 638

Ennen, Edith 594, 705

Erbse, Hartmut 535

Erfurt 24

Erlangen 142, 150 f., 170, 206, 210 f., 213-215, $218,266,274,276,302,339,49^{2}, 5^{27}, 562$, 589,622

Ermatinger, Emil 384

Ermenonville 92

Ernst, Otto 274

Errington, Robert Malcolm 637

Erzgebirge 23, 26, 31

Esch, Arnold 661,705f., 711

Estermann, Monika 18

Estland 281

Euripides 702

Evans, Richard J. 620

Falckenberg, Harald 682

Farinacci, Roberto 302,546

Feldman, Gerald D. 654 f.

Felken, Detlef 310, 594, 662-665, 693 f., 707, $719,73^{6}$

Felßecker, Carl 96

Felßecker, Paul Jonathan 96

Ferdinand II., Kaiser 24

Ferdinand III., Kaiser 25

Fest, Joachim 709

Fichtelgebirge 325

Fick, Katharina $517,57^{2}$

Fick, Roderich 410, 440, $5^{17}$

Finck von Finckenstein, Margarethe 466

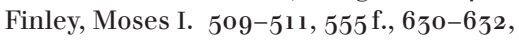
634

Finzi, Aldo 339

Fischer, Hugo 538

Fischer, Joschka 694

Fischer, Jürgen 545, 564-566, $568 \mathrm{f}$., 574, $580-582,584,586,588,605,657,733,735$

Fischer, Otto 259

Fischer, Samuel 258, 330, $33^{2}$

Flach, Dieter 637

Flasch, Adam 211

Flasch, Kurt 692 f., 711

Flashar, Hellmut 702

Fleckner, Uwe 681

Flemming, Alfred 382, 432, 522, 542

Flex, Konrad 282, 469

Flex, Otto 274

Flex, Rudolf 281 
Flex, Walter 273-283, 343, 384, 398, 4.05, $411,469,728$

Fliedner, Theodor 141

Florenz 682, 706

Florescu, Catalin Dorian 686

Fönhus, Mikkjel 4,00, 684 .

Förster, Anna Maria $z^{0}$

Förster, Eusebius 28

Förster, Maria Magdalena s. Beck, Maria Magdalena

Förster-Nietzsche, Elisabeth 299 f., 304

Fontane, Theodor 367,605

Forsthoff, Ernst 396

Foster, Georg 61

Fox, Paula 686

Francke, August Hermann 47, 107

Franco, Francisco 196

François, Etienne 708

Frank, Hans 370, 373, 39o f., 394

Franke, Peter Robert $55^{2}$

Franken 25, 101, 130, 137 f., 144f., 148-150, 172,728

Frankfurt am Main 24, 43, 82, 108, 138, 182 f., 248, 251, 266, 295, 316, 318-320, $3^{6} 5^{\mathrm{f}}, 3^{82}, 428,49^{2}, 494,502,522,53^{2}$, $542,559,567$ f., 580, 584, 598, 602, 605 f., 620-622, 636, 66o, 672,676, 686, 69o, $696,698,706,708,716,73^{2}$

Franklin, Benjamin 586

Frankreich 52, 54, 61, 73 f., 92, 102 f., 115 , $121,134,150,173,179$ f., 192 f., 195, 197, $219,236,263,269,311,314,3^{20}, 3^{22}, 3^{29}$, 345,349 f., $385,399,401,413,45^{2}, 457$, 476 f., 509, 520 f., 533, 544, 632, 634, 652, 679, 700, 705, 708f., 711, 716, 727

Franz Joseph I., Kaiser von Österreich 182

Franzen, Adam Wilhelm 80

Frauenholz, Eugen von 401, 4.05

Frede, Michael 692

Freeden, Eva von 6o1, 6o5-6o7, 613, 645f., $657,659 \mathrm{f}$., $734 \mathrm{f}$.

Frei, Norbert 655,708

Freiburg im Breisgau 158, 211 f., 618, 672, 699,706

Freiburg im Üechtland 524, 561, 692

Freising 270

Freisler, Roland 373, 394

Frevert, Ute 661

Freyer, Hans 504

Freytag, Georg $25^{2}$

Fried, Johannes 690, 706, 708-711

Friedell, Egon 287, $33_{2}^{2}$ f., 343, 345-362, 392, $398,403,417,437,538,564,710,729$

Friedländer, Elli $65^{2}$

Friedländer, Jan $65^{2}$

Friedländer, Max Jakob 4,10
Friedländer, Saul 641, 644, 646, 652-654, 710 f., $73^{6}$

Friedrich II., Kaiser 710

Friedrich II., König von Preußen 32, 35, 89, 408, 709

Friedrich V. von der Pfalz 24

Friedrich, Caspar David 68o, 682

Friedrich, Heinz 481

Friedrich, Johann 172

Friedrich Wilhelm I., König in Preußen 85

Friedrich Wilhelm IV., König von Preußen 137

Friedrichsruh 274

Frisch, Max $73^{2}$

Frobenius, Leo 295 f.

Frobenius, Ruth 295

Fröbel, Julius $153 \mathrm{f}$.

Frost, Robert 688

Fürth 114, 141, 151

Fuhrmann, Horst 593 f., 625, 706

Fuhrmann, Manfred 510

Funkenstein, Amos 64,6

Furtwängler, Adelheid 266

Furtwängler, Adolf 226, 265 f.

Furtwängler, Wilhelm 226, 367

Gaarder, Jostein 693

Gabun 311

Gadamer, Hans-Georg 309f., 399

Gall, Lothar 16, 612,615, 616, 625, 654, 709

Ganghofer, Ludwig $25^{8}$

Garmisch-Partenkirchen 26o, 271

Gauger, Hans-Martin 697

Gay, Peter 6o9, 661

Gaza 688

Geertz, Clifford 705

Gehlen, Arnold 504

Gehrke, Hans-Joachim 637 f., 699, 707

Geiger, Conrad 205

Geiger, Gottlob 256

Geiger, Jakob Eberhard $13^{2}$

Geiss, Immanuel 623

Geisseler, Bernt von 4,19

Gelfert, Hans-Dieter 697, 710

Gellert, Christian Fürchtegott 52, 73, 149

Gelzer, Heinrich 221, $23^{2}$

Gelzer, Matthias 492, 496

George, Stefan 258, 289, 295, 335f., 399, 433, 710

Gerhardt, Volker $69^{2}$

Gervinus, Georg Gottfried 173

Gesner, Johann August Philipp 49f., 86, 110

Geßner, Ludwig 4.04.

Geyer, Karl 179

Ghana 693

Gibbon, Edward 62,633

Giesecke, Michael 14 
Gießen 192, 211, 213, 366

Gilbert, Felix 602

Girgensohn, Karl 260

Giuliani, Luca 683, 711

Gladstone, William Ewart 179

Glarus 65

Glaser, Hermann $5^{27}$

Glauning, Friedrich 264

Gleditsch, Hugo 211, 213,229

Globke, Hans 382, 392-394, 398, 543, 546

Glogau 38

Glum, Friedrich $543 \mathrm{f}$.

Goebbels, Joseph 195, 197, 303, 307, 399, $4,11,437,477$

Gödlin, Anna 65

Göbekli Tepe 703

Göpfert, Rebekka 684

Goerdeler, Carl Friedrich $369 \mathrm{f}$.

Görgel, Wilhelm 205

Göring, Hermann 4,10

Görres, Johann Joseph 294

Göschen, Georg Joachim 38, 42

Goethe, Johann Wolfgang von $57,73,83,109$, 129, 179, 204 f., 243-248, 250 f., 253, 264 , 277, 294, 300, 312, 316, 338, 342, 404, 420, 56o, 573f., 587, 685, 696, 707, 709, 717

Göttingen 46, 235, 303, 508, 535 f., 551, $55^{6}$, $559,566,569,571,573,610,627,643,66$ o, 696,698

Goetz, Hans-Werner 705

Goetz, Walter 307 f., 435

Götze, Albrecht 234

Goldarbeiter, Erwin 351, 353

Goldhagen, Daniel Jonah 653

Goldstücker, Eduard 567

Gombrich, Ernst Hans 529

Goppel, Alfons 518

Gorelik, Lena 655

Gorter, Albert 386

Goschler, Constantin 655

Gotha 51, 129, 255

Gottesgab (Boźí Dar) 23

Goya, Francisco de 682

Grabs, Rudolf 323

Graefe, Axel von 385

Graeser, Wolfgang 293

Graf, Friedrich Wilhelm 644, 695

Graf, Fritz 696

Grant, Michael 554-556

Grass, Günter 477,605

Graupe, Heinz Mosche $65^{\circ}$

Graz 211, 213, 292

Green, Peter 555

Gregorovius, Ferdinand 566

Greifswald 235, 251

Gressius 68
Griechenland 97, 164, 201, 204-208, 210, 212-217, 219-221, 223, 225 f., 228-234, 236, 238 f., 266, 346, 359-361, 423 f., 489 f., 492 , 494 f., $497-501,504,506$,

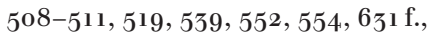
637-639, 683, 700-702, 708, 716

Grillmair, Rolf 482, 519, 540

Grillparzer, Franz 247

Grimm, Gunter 696

Grimm, Jacob 149,248

Grimm, Wilhelm 149, 24,8

Grimmelshausen, Hans Jakob Christoffel von 662

Großbritannien 19, 90, 103, 121, 172, 359, 419f., 490, 457, 519f., 528, 544, 554f., 617, $630,687,705,715$

Grossmann, Atina 655

Grote, George 216, 219

Grote, Gertrud 4.00, 407, 435, $459 \mathrm{f}$.

Grubel, Fred 645, 649

Gruber, Sabine 687

Gruen, Erich S. 555

Gründel, Ernst Günther zoof., 384, 385, $545 \mathrm{f}$.

Gründler, Otto 338 f., 419

Grünig, Ferdinand 386, 404

Grummt, Christina 680

Gruppe, Otto 230

Guayaquil 377

Günsbach 312, 317, 319

Guenther, Johannes von 540

Günther, Siegmund 211, 214 f., $23^{\circ}$

Güntner, Joachim 683

Gürtner, Franz 373

Gütersloh 14,8, 331

Guggenheim, Felix 479

Gundolf, Friedrich 253

Gurjewitsch, Aaron 713

Gutknecht, Christoph 697

Guttmann, Wilhelm 367

Gutzkow, Karl 110

Haarmann, Harald 697

Haarmann, Ulrich 661, 705, 714

Habermas, Jürgen 621-623, 657

Habicht, Christian 535, 698

Hadrian, röm. Kaiser 231, 538, 554

Haegert, Wilhelm 437

Härtel, Gottfried Christoph 314

Haessel, Hermann Adolf 331, 4,19

Hager, Kurt 473

Halle an der Saale $37,44,47,54,108,492$

Halm, Heinz 714

Halm, Karl 185, 209

Hamburg 16, 108, 121, 141, 148, 257, 26o, $323,373,377,492,523,565,573-575,650$, 682,696 f., 709 
Hammer, Caspar 229

Hammerdörfer, Catharina 28

Hammerdörfer, Gabriel 28, 30

Hammerdörfer, Margarethe, geb. Rothen $z^{\circ}$

Hammerdörfer (Küttner), Simon 28

Hammermayer, Ludwig $\mathbf{5 2 6}$

Hamsun, Knut 354

Handke, Peter $73^{2}$

Hannibal 703

Hannover 4,22

Hanser, Carl $55^{\circ}$

Harburg 134

Harder, Richard 492, 494

Harnack, Adolf 235

Harpprecht, Klaus 602

Harris, Arthur 419

Hasinger, Günther 690

Haß, Hans-Egon 558

Hatzfeldt, Paul von 6o4

Hatzfeldt, Sophie von 604

Haupt, Paul 311, 315f.

Hauptmann, Gerhart 274, 710

Hauser, Arnold 528-530, $53^{2}$ f., 538, 546, $570,73^{2}$

Hausmann, Ulrich 502, 698

Havanna 114

Hawaii 690

Haybach, Rudolf 445

Hebbel, Christian Friedrich 24,8

Hebel, Johann Peter 710

Hecker, Friedrich 158

Hedemann, Justus Wilhelm 392

Heerdegen, Ferdinand 211, 213, 229

Hegel, Georg Wilhelm Friedrich 75, 14,2, 174, 201,345

Heger, Hedwig 559

Heiberg, Johan Ludvig 230, 690

Heidegger, Martin 336, 530

Heidelberg 157, 161, 165, 173, 192, 296, 309, $342,366,369,485,495,513,534,536$, $561,610,672,692,698$

Heidenreich, Johann Christoph 36

Heidenreich, Johanna Louise s. Beck, Johanna Louise

Heidenreich, Sophia Jakobina, geb. Vogelgesang $3^{6}$

Heilbronn 80

Heimpel, Hermann 562

Heine, Heinrich 562

Heinrich, Albert 382, 540, 565, 574

Heins, Valentin $374,43^{1}$

Heinze, Richard $33^{\circ}$

Heinzelmann, Anna Susette Katharina, geb. Wöhrle von Wöhrburg 133

Heinzelmann, Babette Lucie Eugenie s. Beck, Babette Lucie Eugenie
Heinzelmann, Christian Gottlieb $121 \mathrm{f}$.

Heinzelmann, Christoph Friedrich 133

Heinzelmann, Johann Georg 133

Heinzelmann, Katharina Magdalena s. Beck, Katharina Magdalena

Heinzelmann, Maria Elisabeth 121

Heisenberg, August 234.

Heißerer, Dirk 697

Held, Hans Ludwig 341, 433

Helsinki 580

Hengstenberg, Ernst Wilhelm 145

Henle, Wilhelm 259

Hennis, Wilhelm 709

Henrich, Dieter 693

Herbart, Johann Friedrich 237

Herbert, Ulrich 622, 653

Herder, Johann Gottfried 155, 247, 562

Herodot von Halikarnassos 499

Herrmann, Wilhelm 235

Hertel, Dieter 702

Hertz, Wilhelm 182

Hertzberg, Arthur 647

Herwig, Johann Justus 54

Herzer, August 186

Herzke, Ingo 687

Herzog, Elisabeth 647

Herzog, Reinhart 700

Hess, Rudolf 404

Hesse, Hermann 274

Heuschele, Otto 292, 315, 349

Heuß, Alfred 503-505, 508f., $55^{1}$ f., 593

Heuss, Theodor $3_{18}$

Heydenreich, Ludwig 528

Heydolph, Georg 54,2

Heydrich, Reinhard 395

Heyse, Paul 182

Hielscher, Martin 664, 684, 686-689

Hierl, Konstantin 404

Hilbrandt, Johann Christoph 4,2

Hildebrand, Klaus 618,622, 625

Hildermeier, Manfred 627

Hillenbrand, Johanna Jakobina 122

Hillgruber, Andreas 622, 625

Himmelheber, Georg 528, 680

Himmler, Heinrich 4,24

Hindenburg, Paul von 368

Hinneberg, Paul 221

Hinrichs, Gustav $211 \mathrm{f}$.

Hinrichs, Johann Conrad 258, 333

Hintze, Otto 292

Hiroshima 466, $53^{1}$

Hirsch, Eike Christian 697

Hirschberg an der Saale 38

Hirth, Georg 169

Hirth, Walter C. F. 294

Hitler, Adolf 196 f., 283 f., $288,298,303,339$, 
343, 351, 354f., 358f., 368 f., 381, 387, 390, 4.02, 4.04, 4.10, 4.12, 417, 4.24, 433 f., 437 f., 463 f., 468, 488, 497, 543 f., 551, 617, 641, $653,709,715$

Höfer, Candida 679

Höffe, Otfried 692, 707

Hölderlin, Friedrich 75, 294, 562

Hölkeskamp, Karl-Joachim 708

Hoeller, Carl 439 f., 519, 540, 547, 571-573

Höllmann, Thomas 714.

Hölscher, Lucian 695

Hölscher, Stefanie 664,681 f.

Hösch, Edgar 705

Hösle, Vittorio $692 \mathrm{f}$.

Hofmann, Johann 234

Hofmann, Johann Christian Konrad 134, 150, 170

Hofmann, Werner 528, 682

Hofmannsthal, Hugo von 294

Hohenaltheim 47

Hohenasperg 64

Hohenemser, Herbert 548

Hohentwiel 81

Hohl, Ernst $5^{02}$

Holberg, Ludvig 579

Holsboer, Florian 691

Holstein 139, 170

Holtfrerich, Carl-Ludwig 654

Holtzbrinck, Georg von 567

Holzberg, Niklas 702

Homer 46o, 63o, 700

Hommel, Fritz 211, 213, 229

Honecker, Erich 473

Hopf, Georg Wilhelm $203 \mathrm{f}$.

Hopmann, Marianne 598

Horkheimer, Max 6zo

Horovitz, Béla 354, 359-361

Hose, Martin 702

Hosius, Carl 232

Hottinger, Heinrich 16o, 177

Hoyerswerda 651

Hrouda, Barthel 638

Huch, Ricarda $293 \mathrm{f}$.

Hübner, Emil 211 f., 228

Hülsen, Christian 229

Hürlimann, Martin 4,13

Humboldt, Wilhelm von 205, 697

Hunger, Herbert $23^{2}$

Husserl, Edmund 504, $53^{\circ}$

Ibsen, Henrik 247

Ihde, Wilhelm 357

Iliffe, John 714

Im Hof, Ulrich 712

Imhof, Arthur E. 618

Indien $100,317,397,586,589,687,694,696$

Innsbruck 211, 213
Inverness 492

Ionesco, Eugène 679

Iran 234, 589, 685

Iriye, Akira 715

Iselin, Isaak 110

Isokrates 4.99

Israel 519, 626, 641-643, 647, 650, 654

Israel, Peter 601

Italien 61, 180, 196, 213, 229, 298, 302, 339, $385-387,401$ f., 4.26, 434, 437, 439, $457 \mathrm{f}$., 518, 528, 533, 552, 539, 545, 597, $633 \mathrm{f}$., $690,705,713,730 \mathrm{f}$.

Jacobsen, Jens Peter 400

Jacoby, Felix 492

Jäckel, Eberhard 646

Jäger, Gerhard 638

Jaeger, Werner 496

Jahn, Adolf 367

James, Harold 654

Janssen, Johannes 270

Japan 194, 586, 711

Jaspers, Karl 309

Jauer 211, 213

Jawlensky, Alexej von 680

Jean Paul 688

Jeanne d'Arc 456

Jeck, Udo Reinhold 693

Jedele, Eugen 256

Jehne, Martin $7^{03}$

Jena $46,5^{\circ}, 56,110,161,221,249,344,400$, $485,487,490$

Jepsen, Alfred 4,1 2, 64,2

Jeremias, Alfred 333

Jerusalem 32, 531, 646, 672,687

Jochsberg 14,4

Johann Friedrich Alexander zu

Wied-Neuwied 81

Johann Georg I. von Sachsen 25 f., 29

Johannes Paul II., Papst 706

Johanngeorgenstadt 26-32, 35, 39

Johnson, Lyndon Baines $5^{19}$

Johnson, Uwe 477

Johst, Hanns 400

Joost, Ulrich 559

Jordan, Ludwig Andreas 170

Joseph II., Kaiser 59-62, 65, 72, 104

Joy, Charles R. 320

Judeich, Walther 229

Jülich 116

Jünger, Ernst 287, 309, 467

Juliana, Königin der Niederlande 467

Jung, Julius 211, 213, 229

Jung-Stilling, Johann Heinrich 579

Junge, Carl 186

Justinian, röm. Kaiser 214, 223, $231 \mathrm{f}$.

Kaddor, Lamya 693 
Kaemmel, Ernst $5^{23}$

Kaerrick, Elisabeth (Less) 293

Kafka, Franz 458, 530, 567, 709 f.

Kahl, Wilhelm 368

Kahrstedt, Ulrich 554

Kaiser, Christian 565

Kaisersheim 55

Kaiserswerth 14,1

Kalifornien 596,646,695

Kaltenbrunner, Ernst 395

Kambodscha 520

Kampfhammer, August 186

Kandinsky, Wassily 258, 680

Kant, Immanuel 39, 46, 59, 109, 178, 214 , 239, 248, 314, 562, 692, 709

Karl der Große, Kaiser 706

Karl Martell 4, 1

Karl Theodor von der Pfalz 81

Karlsruhe 161,682

Kaser, Max 232, 502

Kassel 4,85

Kauders, Anthony 655

Kaufbeuren 121 f., 133

Kauffmann, Gerhard 385

Kaufmann, Vincent $\mathbf{7 2 3}$

Kees, Hermann 234

Kegler, Wilhelm ${ }_{46} 6$

Keil, Ernst $13^{1}$

Kempten 122

Kennedy, John Fitzgerald 322, 519

Kepler, Johannes 334, 54,0, 689

Kermani, Navid 714

Kerschensteiner, Georg $\mathbf{2 3} 6$

Kiaulehn, Walther 466, 477-479, 546

Kiel 211-213, 251, 495f., 535f., 551, 566, 573

Kielmansegg, Johann Adolf Graf 618

Kiesel, Helmuth $\mathbf{5 2 5}$

Killy, Walther $537 \mathrm{f}$, 546, 556-564, $566 \mathrm{f}$, $57^{1-574}, 594,63^{2}, 644,658,672,73^{2}-734$

Kinski, Klaus $5^{18}$

Kinzel-Amuser, Ingrid 6oo-6o2, 606

Kippenberg, Anton 581

Klaffenbach, Günther 485, 492

Klagenfurt 292, 687

Klages, Ludwig 294-297, 334

Klein, Tim $337 \mathrm{f}$.

Kleinasien 703

Kleist, Heinrich von 181, 247, 270, 341, 563 , 710

Klemke, Werner 546

Kleopatra, ägypt. Königin 555

Kleßmann, Christoph $53^{2}$

Klingner, Friedrich 492

Klopstock, Friedrich Gottlieb 53, 205

Knorr, Julius $\mathbf{1 6 9}$

Kober, Adolf 246
Koblenz 158, 251

Koch, Bernhard 466

Koch, Erich 463

Koch, Franz 560

Koch, Friedrich $25^{1}$

Koch, Friedrich Wilhelm 386, $523 \mathrm{f}$.

Koch, Hans-Albrecht 701

Koch, Max 251

Kocka, Jürgen 591 f., 6o9, 617, 623

Köln 226, 246, 492 f., 502, 513, 611, 622, 645, 714

Königsberg 249, 462 f., 466, 468, 551, 56o

Königseder, Angelika 64,

Könnecke, Erich 565

Koeppen, Wolfgang 662

Körner, Theodor 273, 281

Köster, Albert $\mathbf{3 2 5}$

Kohl, Helmut 622, 644, 647

Kohl, Karl-Heinz 705

Koktanek, Anton Mirko 308-310, 546

Kolb, Frank 703

Kongo 311

Konstantin I., röm. Kaiser 231, 634, 637, 647, 702

Konstantinopel 538

Konstanz 102, 163, 700, 714

Koopmann, Helmut 709

Kornemann, Ernst 296, 492 f., 508

Kornhardt, Hildegard 305 f., 308

Koselleck, Reinhart 15

Kotab, Herma 351, 361

Krämer, Gudrun 707

Krafft, Christian 14,2, $15^{\circ}$

Kraft Ernst zu Oettingen-Wallerstein 45, 54 f., $72,82,96,114$

Kramp, Willy 446, 465

Kraus, Andreas 527

Krautheimer, Richard 680

Kreisel, Heinrich 528,680

Kreutziger-Herr, Annette 717

Krieger, Arnold 467

Kristof, Nicholas D. 715

Kromayer, Johannes 234

Kronenberg, Moritz 248

Krueger, Felix 337

Krüger, Gustav $23^{2}$

Krüger, Herbert 523

Krüger, Lore 585

Krüger, Michael 683

Kruft, Hanno-Walter 680

Krug, Antje 698

Krumbacher, Karl 20, 208, 211,214, 220-223, 226, 232, 234, 261-263, $265-267,496$

Krupp von Bohlen-Halbach, Bertha 272

Kuba 322, 519 
Kubitschek, Wilhelm 229

Kühn, Manfred 709

Küster, Hansjörg 689

Kufstein $35^{1}$

Kumpmann, Walter 643

Kunisch, Johannes 709

Kunkel, Wolfgang 503

Kurbayern 115

Kurpfalz 81 f., 116

Kursachsen 24f., 53

Kurzal, Andreas 666

Kurzke, Hermann 709

Laband, Paul 366,376

Lachmann, Karl 335

Lagarde, Paul de 347

Lai, Whalen 695

Lambarene $3^{11}, 3^{1} 5^{-3^{1}}, 3^{19}, 3^{21}, 3^{23}, 43^{6}$

Lambrecht, Karl $325 \mathrm{f}$.

Lampert, Ludwig 266 f.

Landau 158

Landerer, Johann Jakob 50

Landmann, Robert von 176

Landry, Harald 292, 299

Lang, Georg Heinrich $47 \mathrm{f}$.

Lang, Karl Heinrich von 45, 47

Lang, Ludwig $13^{6}$

Lang, Thomas 687

Lange, Tönjes 380

Langemarck 283

Langen, Albert 258, 272, 414

Langenfaß, Friedrich 338

Laporte, Luise (Lu) 4.13, 4.15, 445, 448, 452 f., $455 \mathrm{f}$., 460

Larenz, Karl 396

Larfeld, Wilhelm $228 \mathrm{f}$.

Lassalle, Ferdinand 174, 218, 604

Last, Hugh 499

Latte, Kurt 502

Latude, Henri Masers de 585

Lausanne 317

Laveleye, Émile de 179

Layriz, Friedrich $149 \mathrm{f}$.

Le Goff, Jacques 693, 711-713

Le Suire, Georg Daniel 82

Le Suire, Georg Wilhelm 82

Le Suire, Johann Wilhelm 82

Lehmann, Gustav Adolf $702 \mathrm{f}$.

Lehmann, Niclaus 186

Lehmann, Paul 223

Lehndorff, Carl Meinhard von 468

Lehndorff, Hans von 461-471, 483, 546

Lehndorff, Heinrich von 4,68

Lehndorff, Siegfried von 462

Leibniz, Gottfried Wilhelm $293 \mathrm{f}$.

Leipzig 24, 37-40, 43 f., 48, 51-53, 56 f., 64, 73, 108f., 113, 127, 131, 181, 211, 213, 221,
234, 251 f., 255, 260, 270 f., 290 f., 297,

306, 314, 317, 325, 329, 331, 333, 337, 341,

$354,369,383,407,415,419,423,447$,

488 f., $49^{6}$ f., 517, 551, 579-581, 583-585,

$587 \mathrm{f} ., 642,654,682,686,711$

Leistner, Yrsa von 471

Lenin, Wadimir Iljitsch 298

Leningrad 470

Lent, Ingrid 680

Lenz, Hermann 477

Lenz, Siegfried 477

Leonhardt, Jürgen 703

Leonidas I., König von Sparta 499

Leppin, Hartmut 702

Lepsius, M. Rainer 621

Lerche, Peter 396

Lesage, Alain-René 579

Lessing, Gotthold Ephraim 37, 205, 346, 562, 585,709

Lessing, Theodor 260, 297, 346

Lessing, Theophilus 37

Lesskow, Nikolai Semjonowitsch 292 f., 540, 684

Leumann, Manu 234

Leupold, Dagmar $686 \mathrm{f}$.

Leuschner, Wilhelm 617

Levi, Löw 105

Levin, Julius 293

Lewes, George Henry 245

Lewinsky, Tamar 655

Leydel, Wilhelm 146

Lichtenberg, Georg Christoph 559 f., 662

Liebermann, Max $366 \mathrm{f}$.

Liebmann, Hilde, geb. Mayer $367,376-378$

Liebmann, Irma 366 f., 378

Liebmann, Karl 365

Liebmann, Karl Wilhelm 366 f., 371 f., $374-380,43^{2}$

Liebmann, Lili Fanny, geb. Herxheimer 366 , 378

Liebmann, Margarete $366 \mathrm{f} ., 378$

Liebmann, Otto 19, 341, 365-376, 378-382, $389,392,394$ f., 409 f., 414, $73^{0}$

Liebmann, Wolfgang $367,376-378$

Liedloff, Jean 691

Liège 179

Linden, Walther 24,4, 385

Links, Roland 579, 581 f., 584

Lissabon 708

Litt, Theodor 236

Loeb, James $265 \mathrm{f}$.

Löbell, Frank 4,23

Löhe, Gottfried 186

Löhe, Wilhelm 134, 14,1-149

Loew-Cadonna, Martin 458

Loewenheim, Francis 603 
Löwenstein 113

Löwith, Karl 536, 547

Lolling, Habbo Gerhard 211, 213, 229

London 121, 359f., 459, 492, 60o, 681

Loos, Lina 347

Lorck, Carl von (Klaus Klootboom-

Klootweitschen) 468

Los Angeles 646

Losemann, Volker 633

Lotar, Peter 320

Lothringen $236,275,326$

Lotzbeck, Christian Albrecht 49

Lotze, Detlef 716

Luchterhand, Heinz 380

Luchterhand, Hermann 380

Luckmann, Ilse 452-454

Ludwig I., König von Bayern 131, 136, 157, $257 \mathrm{f}$.

Ludwig XVI., König von Frankreich 54, 103

Ludwig zu Oettingen-Wallerstein 137

Ludwig Eugen von Württemberg 102

Ludwigsburg 177,322

Lübbe, Hermann 310

Luhmann, Niklas 13f., 668

Lund 492

Lunéville 115

Luther, Martin 24-26, 3o, $3^{2}$ f., 37, 42, 47, 49, $114,118,131,134,141 \mathrm{f} ., 144-151,158$, 203, 235, 270, 338 f., 462, 549, 562, 657, 710,727

Luxemburg 162

Lykurgus 205

Lynch, Benito 400

Maas, Paul 403

Maastricht 708

Maaz, Hans-Joachim 691

MacGregor, Neil 715

Machfus, Nagib 586

Machiavelli, Niccolò 602, 710

Mackowiak, Klaus 697

Madeja, Michael 691

Madrid 681

Mähren 24

Märtl, Claudia 717

Maetzig, Kurt 472

Magdeburg 28

Maier, Bernhard 700, 705

Maier, Hans 569, 708, 710

Mainz 365, 538, 630, 634

Makedonien 637

Malitz, Jürgen 718

Mallarmé, Stéphane 399

Mallmann, Walter 391, 432, 435, 439

Malraux, André 527

Mandelkow, Karl Robert 696

Manitius, Max 223, 232
Mann, Golo 540, 599

Mann, Heinrich 709

Mann, Thomas 257, 287, 300, 336, 377, 446, 457,709

Mannheim 16,81

Mannheim, Karl 529

Manz, Friedrich 186

Marathon 404

Marbach 672, 710

Marbach, Othon $5^{21}$

Marburg 211, 213, 337, 633f., 637

Marc, Franz 680

Marcion 354

Marcus Antonius $51^{12}$

Marek, Christian 703

Marek, Kurt Wilhelm (C. W. Ceram) 595

Marg, Walter 494, 496, 538f., 603

Maria Theresia, Erzherzogin von Österreich 60

Markschies, Christoph 695, 709

Marokko 288

Marquardsen, Heinrich 170

Martin, Emmy 312, 314

Martin, Kurt 527, 546

Marx, Karl 153, 529, 584, 597, 630

Marr, Abraham $105 \mathrm{f}$.

Massachusetts 646

Matthias, Adolf 209, 237 f., 248, 252, 265

Matz, Friedrich 496

Maul, Stefan M. 704

Maunz, Theodor $396,547 \mathrm{f}$.

Mauser, Siegfried 716

Mayer, Gustav 6o4

Maximilian Christoph von Rodt 102

Maximilian I. Joseph, König von Bayern $116 \mathrm{f}$.

Maximilian II. Joseph, König von Bayern 157 , 325

Mazower, Mark 715

Mecklenburg 292, 469

Mehring, Reinhard 710

Meier, Christian 509, 512, 639,642, 645f., 648,698

Meier-Graefe, Julius 476

Meinau, Caroline 119

Meiner, Felix 317, 323

Meister Eckhart 693

Melanchthon, Philipp 127, 142, 201, 206

Melville, Herman 688

Mendelssohn, Moses 65o, 662

Menz, Gerhard 272

Menzel, Adolph von 368

Merck, Johann Heinrich 245

Merian, Matthäus 41

Merkelbach, Reinhold 554

Merle, Robert 476

Messerschmidt, Manfred 618f., 624 
Mette, Hans Joachim 387

Metz-en-Couture 326

Meunier, Constantin 366

Meyer, Arnold Oskar 385

Meyer, Carl Joseph 121

Meyer, Eduard 216

Meyer, Ernst 509

Meyer, Johann Mathias 149

Meyer, Michael A. 646, $649 \mathrm{f}$.

Meyer, Richard Moritz $247 \mathrm{f}$.

Meyer, Stephan 4o6, 689

Meynier, Johann Heinrich 119

Michelangelo Buonarroti 710

Michigan 147

Mielsch, Harald 698

Miller, Johann Martin 100

Millin, Sarah Gertrude 384

Miltiades 499

Mindelheim 503

Minder, Robert 547

Mitteldeutschland 43 f., 215

Mittenzwey, Kuno (Kuno Renatus) 302

Mitterauer, Michael 710

Mittermaier, Wolfgang 369

Möller, Horst 623,625

Moeller van den Bruck, Arthur 293, 302

Mölln 651

Mönchsroth 134

Mörike, Eduard 269, 686

Mohilowsky, Albertus 105

Mohl, Robert von 157, 165

Mohler, Armin 309

Molière, Jean-Baptiste Poquelin 247

Moltke, Freya von 697

Moltke, Helmuth James von 697

Momigliano, Arnaldo 552, 633 f., 637

Mommsen, Hans 464, 504, 622

Mommsen, Theodor 186, 214, 219, 222, 238, 493 f., 504, 509

Mommsen, Wolfgang J. 622

Monath, Georg Peter 48

Mongolei 586

Montez, Lola 157

Montgelas, Maximilian von $5 \mathbf{5 2 6 , 7 0 9}$

Montrouge 4,01

Moosbauer, Günther 702

Moras, Joachim 521, 538

Moreau, Jean-Victor 75

Moritz, Karl Philipp 586, 662

Moser, Albert 186

Moser, Johann Jacob 8 of.

Moskau 668

Mossé, Claude 555

Mosse, Rudolf 272

Mozart, Wolfgang Amadeus 113, 688

Muegel, Oskar 366
Müller, Albert von $43^{1}$

Müller, Barbara 29

Müller, Ernst von 34,2

Müller, Eva s. Beck, Eva

Müller, Georg 249, 272, 4,14

Müller, Iwan von 210-214, 224f., 227, 230, $233,236,262,267$

Müller, Johannes 26o, 276, 4,26, 4,29 f., 434

Müller, Klara, geb. Beck $133 \mathrm{f}$.

Müller, Ludwig (Lehrer) $133 \mathrm{f}$.

Müller, Ludwig (Bischof) 34,0

Müller, Rabeya 693

Müller, Samuel 29

Müller, Wilhelm 709

Müller-Karpe, Hermann 511, 524, 568, $63^{8}$

München 16, 19, 45, 52, 113 f., 116 f., 122 , $138 \mathrm{f} ., 153,156-158,160 \mathrm{f} ., 165,169,171$, $176,181,185,187,189$ f., 195, 208-211, 213-215, 218, 220-222, 225-228, 231, 233, $236,247,249,251,255^{-259,265,270-274,}$ 281 f., 287 f., 290 f., 294-296, 298 f., 304 , $312-315,325-327,329,336-338,341,343$, 357-362, 369, 37 1, 380, 382, 385 f., $388 \mathrm{f}$., 392, 394, 398f., 405, 413-417, $419 \mathrm{f}$., $422-425,427-431,433,435,437,449,45^{1}$, 453 f., 456, 458 f., 462, 466 f., $472,474-477$, 479 f., 485 f., 488 f., $492-497,502,507$ f., $513,5^{16-519}, 5^{25}, 5^{29}, 539-54,2,544$, 547 f., 559 f., 565,567 f., $570,579-581$, 583, 586, 592 f., 596, 598-6oo, 6ogf., 615, 620, 623 f., 626 f., 639, 641-647, 649, 651 f., 654 f., 661, 670, 672-674, 676 , 681-688, 691, 695-697, 699, 702, $711 \mathrm{f} .$, 716,728

Münster 494, 592,601, 6o7, 614, 629 f., 636, $662,695,706$

Münter, Gabriele 258

Münzer, Thomas 97

Muhammed $492 \mathrm{f}$.

Mundbach, Georg Gottfried 35, 42, 77, 111

Murray, Gilbert 499

Murray, Oswyn $55^{2}$

Muschg, Adolf 686

Muschg, Walter 336

Mussolini, Benito 298, zo1 f., 338, 385-387, 411, 543, 729

Mussolini, Vittorio 302, 402, 546

Nablus 688

Nagasaki 531

Nagel, Tilman 589,714

Nancy 566

Napoleon I. Bonaparte, Kaiser der Franzosen $115^{-117}, 119,204,405,614,667,709$

Napoleon III., Kaiser der Franzosen 162

Nassau 100

Neidhart, Carl Vollrath $118 \mathrm{f}$. 
Neresheim 55

Netz, Reviel 69o

Neuburg 157, 174, 413

Neuendettelsau 14,1 f., 149

Neuwied 81, 100, 251

New Haven 492

New York 317, 597, 630, 645, 686, 688

Newald, Richard 524, 556, $55^{8}$

Nicolai, Friedrich 37 f., $45,60,80,84$, 108-110, 120, 127

Nicolaus Cusanus 692

Niderlechner, Max $379 \mathrm{f}$.

Niebuhr, Barthold Georg 153, 205

Niederbayern 64,87

Niederlande 24, 61, 177, 367, 384, 411, 467, 561

Niemeyer, Max 258

Niese, Benedictus 211, 213, 230, 232, 502

Niethammer, Friedrich Immanuel von 158 , 201

Nietzsche, Friedrich 247 f., 260, 277, 288, 294, 297, 299 f., 304, 312, 334, 335f., 338 346 f., 397, 692, 729

Nilsson, Martin Persson 232, 234, 489, 492, 495

Nippel, Wilfried 710

Nipperdey, Hans Carl 611

Nipperdey, Thomas 6oz,6o9-616, 618-626, 642,645 f., $650,710,733$

Nipperdey, Vigdis 615

Nisbet, Hugh Barr 709

Nissen, Heinrich 211 f., 228

Nock, Arthur Darby 49o, 494

Noel, William 690

Nördlingen 18f., 31, 35 f., 39-42, 44-49, $5^{1-53}, 55^{-57}, 60,63,65,67-72,74$ f., 77 , 81-87, 90-92, 94-100, 102-120, $122 \mathrm{f}$., $127-139,144-146,148 \mathrm{f}$., 151, 155-159, $162,167,169-174,177-179,181,184-187$, 193, $211,243,255$ f., 258, 269-272, 305, $325,329,33^{2}, 411$ f., 414, 422 f., 425, 427, $437,447,45^{1}, 5^{15}-5^{18}, 541$ f., 565, 674 , 726,728

Nördlinger Ries 4,1 f., 48, 53, 74, 106, 136 f., $174,412 \mathrm{f}$.

Nohlen, Dieter 692

Nolde, Emil 680

Nolte, Ernst 597, 622 f., 625

Nolte, Paul 627

Nolte, Ulrich 664, 695

Nopitsch, Christoph Friedrich Wilhelm 94

Nora, Pierre $708 \mathrm{f}$.

Nordamerika 90, 141, 145-151, 310, 582, 626

Norddeutschland 24, 38, 43 f., 53, 56, 145 , 171,215

Nordhausen 108
Norwegen 337, 354

Nouts, Michiel 410

Novalis (Friedrich von Hardenberg) 562, 709

Nürnberg 48, 54-56, 82, 96, 110, 128, 133 , 137, 142, 144, 149, 158, 186, 201, 203, 205 f., 211 f., $264,375,392,398,527$

Oberaudorf am Inn 4,20

Obenauer, Karl Justus 386

Oberlausitz 24

Oberpfalz 24, f., 87

Oberschwaben 64

Oberzell 185

Oehmichen, Gustav 230

Oermann, Nils Ole $\mathbf{3 2 4}$

Oertel, Friedrich 219

Österreich 24, 35, 38, 43 f., 53, 6o f., 64, 87, 89 f., $135,149,161,170$ f., 182 f., $195^{-197}$, $261,292,351,354-356,35^{8-360,368,}$ $399,445^{-448}, 45^{2-454}, 45^{6}, 459,580$, $583,666,687,690$

Oestrich 558

Oettingen 40, 100, 133

Oettingen-Wallerstein 4.5 f., 50 f., 63, 94 f., 116,120

Oexle, Otto Gerhard 723

Ohio 146

Oldenbourg, Rudolf $5^{18}$

Oldenbourg, Wilhelm 291 f., 4,23, 4,25

Oncken, Wilhelm $192 \mathrm{f}$.

Onken, Julia 691

Orelli, Heinrich von 177

Orsenna, Érik 711

Oslo 318, 320, 359

Osmanisches Reich 90

Osterhammel, Jürgen 663, 705, 714 f., 736

Ostrogorsky, Georg 234

Ott, Claudia 589

Otto, Gertrud, geb. von Sprockhoff 486, 492

Otto, Rudolf 337,341

Otto, Walter 233-235, 240, 485 f., 490, 492, 495,499 f., $502,507,513$

Oxford $398,403,492,499,554,592,598$, $600,602,613,646,711$

Paeschke, Hans 538

Pahl, Johann Gottfried 52 f., 74-76, 119

Pakistan 589

Palästina 234, 351, 643, 687

Palandt, Otto 395

Panzer, Friedrich 24,8f.

Papen, Franz von 387

Pappenheim, Wilhelm Graf zu 54

Paret, Peter 602

Paris 92, 103, 135, 179, 189, 192 f., 197, 263, $317,325,365,377,385,400,413,448,453$, $492,510,519,527,681,711$

Parrot, André 528 
Partsch, Susanna 693,717

Parzinger, Hermann 703

Pasing 195

Passau 128

Patzig, Günther 692

Paulsen, Friedrich 236

Pauly, August Friedrich 209

Pavia 634

Payr, Bernhard 410

Pechel, Rudolf 292, 303

Perikles 554, 702

Perowne, Stewart 538, 554

Perthes, Friedrich Christoph 108, 121, 129, 272, 727

Pettenkofer, Max von 257

Pfalz 159, 170, 551, $57_{1}$

Pfeiffer, Gerhard 527

Pfeiffer, Rudolf 398,638

Pfeil, Georg 177

Pflanze, Otto 709

Philipp II., König der Makedonen 492 f., 499, $5^{13}$

Philipp Carl Joseph zu Oettingen-Wallerstein 45

Pick, Behrendt 229

Pierro della Francesca 706

Pietsch, Ursula 553, 555

Piganiol, André 492

Pillau 466

Pindar 702

Pinthus, Kurt 567

Piper, Klaus 679

Piper, Reinhard 272, 293, 307, 417, 428, 435

Pirckheimer, Willibald 397

Pirenne, Henri 6zo

Pirna $3^{2}$

Pitt der Ältere, William $45^{1}$

Pius II., Papst 706

Planck, Karl Christian $177 \mathrm{f}$.

Platen, August von 367

Platthaus, Andreas 717

Platon 494, 499

Platten (Horní Blatná) 23, 26, 28

Plautus 251, 550, 553

Pleschinski, Hans 688

Plinius der Ältere 226

Plotin 494, 538

Poe, Edgar Allan 710

Pöhlmann, Johannes Paul 119

Pöhlmann, Robert von 211, 213, 215-220, 229,232 f., 499,513

Pöls, Werner 591

Pohl, Dieter 653

Polen 102 f., 405, 413, 457, 469, 6o6, 668, 714

Polidori, Robert 679

Pommern 469
Pompeius Magnus, Gnaeus 703

Popitz, Johannes $366, z_{69} \mathrm{f}$.

Poppin, Anna Margaretha 70

Portugal 317

Poseidonios 336

Posen ${ }_{46} 64$

Possenhofen 304

Potsdam 287, 368, 4,28

Pousseur, Henri 547

Prag 24, 186, 211, 213, 473f., 554, $65^{2}$

Prantl, Heribert 670

Preuß, Hugo 166

Preußen 32, 35, 37, 87, 89, 139, 161 f., 165, 169-171, 180, 183f., 193f., 214, 226, 237, 259, 264, 272, 275, 287-290, 294, 298, 302, 327, 331, 340, 369, 373, 395, 446, 461-471, 482 f., 493, 564, 601, 6o3-6o5, 618, $703 \mathrm{f}$.

Princeton 6oz,663,691, 693

Prinz, Friedrich 527

Prölss, Erich 547

Puschkin, Alexander 684,

Pustet, Friedrich 128

Quito 378-380

Raabe, Wilhelm 568

Rader, Olaf B. $709 \mathrm{f}$.

Radkau, Joachim 690

Raeber, Kuno 476

Ranke, Heinrich 144

Ranke, Leopold von 189 f., 205, 219,294 , $346,504 \mathrm{f}$.

Rath, Ernst Eduard vom 196

Raulff, Ulrich 710 f., 716

Ravensburg 105

Rávic Strubel, Antje 688

Raw, Johann Philipp 144.

Reagan, Ronald 64,4

Rebenich, Stefan 717

Redeker, Konrad 395

Redenbacher, Wilhelm 144

Reemtsma, Jan Philipp 653, 709

Rees, Matt Beynon 687

Regensburg 31, 35f., 38, 42, 6o, 115, 517, $5^{26}$

Rehlingen, Johann Baptist von 63, 67

Rehm, Albert 492

Reich, Philipp Erasmus 38, 43, 52

Reichard, Heinrich August Ottokar $5^{\mathbf{1}}$

Reifferscheid, August 211

Reifferscheid, Eduard 380

Reimer, Georg 295

Reims 326

Reindel, Kurt 527

Reiners, Ludwig 337, 406-408, 419, 422, 433, 538,540

Reinhard, Wolfgang 706, 710

Reinhardt, Fritz 386

Reinhardt, Karl 336 
Reinhardt, Max 345, 351

Reinhardt, Volker 705, 710

Reinharz, Jehuda 64,6

Reinsberg, Carola 698

Remarque, Erich Maria 273

Rembrandt van Rjin 682

Renatus, Kuno 385

Reut-Nicolussi, Eduard 4,02

Rheinland 55, 196

Rhodes, Cecil 298

Richarz, Monika $645 \mathrm{f}$.

Richter, Hermann Michael 183

Richter, Otto 211, 213, 229

Richtscheid, Hans 455, 501, 515, $5^{21}$ f., 529, 532, $535^{f ., ~ 538-541, ~} 543^{f .,} 55^{\circ}, 567,57_{1}$

Riedel, Emil 176

Riehl, Wilhelm Heinrich 184

Riemer, Peter 702

Rilke, Rainer Maria 258

Ritschl, Albrecht 235

Ritter, Gerhard A. 591-593, 598f., 6o1, 6o9, 710,733

Robert, Louis 485,492

Rockefeller, John Davison 534

Rodenwaldt, Gerhart 226, 496

Roeck, Bernd 706

Röd, Wolfgang 692

Röhl, John C. G. 6o6, 709

Röhm, Ernst 389

Röhrig, Ferdinand 136

Rösener, Werner 712

Rohde, Hanna 4,10

Rohlfs, Gerhard 4,28

Rohmer, Emil 187

Rohmer, Ernst 20, 151, 158-164, 166 f., $169-187,189-194,201,255,258,261,264$, $269,428,573,724$ f., 727 f., $73^{1}$

Rohmer, Eugen 187

Rohmer, Friedrich 153, 154-163, 166 f., 174, $177,183,189,193,728$

Rohmer, Friedrich Theodor Georg Eugen 159

Rohmer, Gustav 187, 412, 425, 429, 431

Rohmer, Mathilde 159

Rohmer, Sophie, geb. Planck 158

Rohmer, Johann David 158

Rohmer, Theodor 153, 154f., 158-16o, 163, $167,177,189$

Rohmer, Theodor (Sohn von Ernst Rohmer) 187

Rom 24, 41, 62, 153, 182, 196, 201, 205-207, $212-214,216,219,223,226,228-234,239$, $4^{28}, 490,494,497,499-504,506-509$, 511, 554, 566, 629f., 632-635, 637 f., 68o, 682,695,698-703, 705f., 708, 711, 715

Romanos 222

Roscher, Wilhelm 165
Rose, Hans 399

Rose, Herbert Jennings 554

Rosenberg, Alfred 401, 494, 560

Rosenberg, Leo 366, 374, 378, $43^{2}$

Rosner, Captain $\mathbf{4 2 5}$

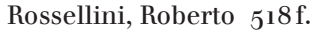

Rostock 150, 64,2, 651

Rostovtzeff, Michael 492, 494, 5o8, 633

Roth, Christoph zo $^{\circ}$

Roth, Karl Ludwig 142, 201-208

Roth, Joseph 365

Rothenburg ob der Tauber 49, 186

Rothermund, Dietmar 714

Rotteck, Karl von 164

Rousseau, Jean-Jacques 92

Rowohlt, Ernst 258, $563 \mathrm{f}$.

Rubens, Peter Paul 683

Rudolf II., Kaiser 24

Rüegg, Walter 704f.

Rüpke, Jörg 696

Rürup, Reinhard 649

Ruh, Kurt 696

Ruhrgebiet 257

Rumänien 668, 679

Runciman, Steven 538,591

Ruppert, Wolfgang 618

Rupprich, Hans 559

Russland 90, 105, 175, 298, 301, 337, 445, 470,485 f., 541, 597, 627, 667 f., 674, 713

Rust, Bernhard 403

Rutter, Pfarrer 105

Saaremaa (Ösel) 281, 283

Sabrow, Martin 709

Sachs, Ernst 379

Sachsen 23, 26, 28-32, 35, 37, 43, 56, 386, $389,496,587,651,675$

Sachsenhausen 377

SAID $685 \mathrm{f}$.

Saint Quentin 326

Salles, Georges $5^{27}$

Salzburg 64, 108

Samos 261

Samuel, Alan Edouard 229

San Nicolò, Mariano 227, 492, 502

Sander, Heinrich $60 \mathrm{f}$.

Santayana, George 400, 547

Sapper, Agnes 208

Sartorius, Carl 259, 522

Sauer, Bruno 231

Sauer, Robert 548

Sauerbruch, Ferdinand von 471

Sauerländer, Willibald 528,683

Savelkouls, Hermann $387 \mathrm{f}$.

Savigny, Friedrich Carl von 153

Schachermeyr, Fritz 485

Schanz, Martin von 211, 214, $231 \mathrm{f}$. 
Schäffle, Albert 165

Schäufelin, Hans Leonhard 122

Scharff, Alexander 4.92

Schattenmann, Johannes 535

Scheffau 423

Scheffel, Helmut 547

Schefold, Karl 546

Scheiner, Otto 342

Schelling, Friedrich Wilhelm Joseph 292, 300, 397

Schemm, Hans $195 \mathrm{f}$.

Scherer, Wilhelm 243

Scherf, Walter 697

Schertel, Josephine Johanne s. Beck, Josephine Johanne

Schieder, Theodor 464, 591, 616

Schiefer, Albert Thilo 186

Schiewe, Jürgen 406

Schiffer, Eugen 368

Schiller, Friedrich 73, 121, 193, 204f., 243 , 247,709

Schiller, Hermann 211, 213, 230

Schiller, Walter 585

Schilling, Heinz 710

Schimmel, Annemarie 589, 710

Schirnding, Albert von 4.07

Schlak, Stefan 709

Schlecht, Raymund 134

Schleiermacher, Friedrich 14,2, 151, 153

Schlesien 135, 213

Schleswig 139, 170, 251

Schlieffen, Alfred von 326

Schlingensiepen, Ferdinand 709

Schlosser, Karl 194

Schmalz, Josef Hermann 211, 213, 229

Schmarsow, August 326

Schmid, Alois 527

Schmid, Wilhelm 231 f., 4,23, 4.27, 4,89

Schmid, Richard 538

Schmidt, Christiane 664, 685

Schmidt, Friedrich Christian 55

Schmidt, Harald 6o9, 621, 627

Schmidt, Helmut 661, 694, 719

Schmidt, Klaus 703

Schmidt, Ludwig 388 f., 4,02

Schmidt, Melchior 28

Schmidt, Michael Ignaz 54

Schmidt, Peter Lebrecht 700

Schmidt-Dengler, Wendelin 458

Schmidt-Glintzer, Helwig 714

Schmidtler, Michael 4,17

Schmitt, Carl 368f., 373, 375 f., 381, 389 f., 394, 402, 404, 710

Schmitt, Eberhard 634

Schmitt, Hatto H. 551

Schmitthenner, Paul 401
Schneider, Carl 538f., 551, 637

Schneider, Hermann 403, 431, 455

Schneider, Walther 361

Schnitzler, Arthur 252, 377

Schiwy, Günther 532, 657, 709

Schöne, Albrecht 16, 107 f., 558-561, 566 f., $571 \mathrm{f}$., $660 \mathrm{f}$.

Schönemann, Lilli 24,4

Schönermarck, Johann Christoph von 95

Schönfelder, Heinrich 340, 381, 389, 515, 522

Schöpperlin, Johann Friedrich $46,49,53,86$, 118,127

Scholl, Hans 641, 653, 697

Scholl, Sophie 641, 653,697

Scholz, Otto Heinrich 380

Schopenhauer, Arthur 260

Schoske, Sylvia 681

Schostack, Renate $\mathbf{3 8 2}$

Schramm, Percy Ernst 16

Schröpel, Karl 269, 305, 329, 4,12, 427, 432, 543,565

Schröter, Manfred 291 f., 296, 308 f., 334

Schubart, Christian Daniel 64

Schubart, Ludwig 64

Schubart, Wilhelm 228

Schünemann, Peter 581

Schütte, Stefanie 717

Schullze, Erich 431

Schultheß, Heinrich 16o, 177, 189-191, 193 f., 198, 384, 397, 728

Schultze-Westrum, Edith 518

Schulz, Gerhard 525, 562, 709

Schulz, Heinrich 4,12

Schulze, Friedrich 169

Schulze, Hagen 707 f., $712 \mathrm{f}$.

Schulze, Winfried 504, 620

Schulze-Delitzsch, Hermann 174

Schumacher, Alexandra 664, 682

Schwab, Gustav $201 \mathrm{f}$.

Schwaben 40, 47, 74-76, 119, 121, 123, $133 \mathrm{f}$., $138,145,172,174,180,413,723,726,728$

Schwäbisch-Gmünd 186

Schwäbisch-Hall 470

Schwartz, Gary 682

Schwarzenberger, Major $\mathbf{3 2 7}$

Schweden 234, 339, 495

Schweitzer, Albert 311-324, $33^{2}$ f., 343, 349, $39^{2}, 397,43^{6}, 5^{17}, 5^{19}, 53^{0}, 549,564$, 570 f., 729

Schweitzer, Johann Baptist 392, 517, $57^{2}$

Schweitzer Bresslau, Helene 311, $316 \mathrm{f}$.

Schweitzer Miller, Rhena 317

Schweiz 20, 23, 60, 102, 114, 128, 135, $153-156,160,164$ f., 177, 234, 293, 306, 316, 323 f., 334, 336, 353 f., 356, 384, 414, $476,496,5^{24}, 580,583,666,668,686,696$ 
Schwerte 465

Schwickert, Engelhard Benjamin 38, 109

Schwink, Walther 438

Schwyzer, Eduard 232, 234

Sedan 191

Seebold, Elmar 561

Seibt, Gustav 661, 709, 711

Seidl, Erwin 492

Seifert, Alwin 479,689

Seldte, Franz 404, 546

Seling, Helmut 680

Sen, Amartya 663,694, 736

Senckel, Barbara 691

Senefelder, Alois 122

Septimius Severus, Lucius, röm. Kaiser 555

Seyerlen, Rudolf 160 f., 163

Shakespeare, William 129, 247, 346

Shaw, George Bernard 4,21

Shedletzky, Itta 645

Sheehan, James J. 609

Shirer, William Lawrence 544

Sholem, Betty 645

Sholem, Gershom 645

Siebeck, Oskar 314

Siebeck, Paul 313f., 323

Siedler, Wolf Jobst 610,648

Signorelli, Luca 180

Simmel, Georg 253, 529, 723

Simon, Heinrich $5^{87}$

Simon, Marie 587

Simor, Susanne 663

Sinn, Ulrich 702

Sittl, Karl 226, 231, 262, 267

Sizilien 457, $63^{2}$

Skandinavien $337,353,384,400,413$

Skeat, Theodore Cressy 492

Skrjabin, Elena 470

Slevogt, Max 367

Snell, Bruno 535

Snyder, Timothy 715

Sokrates 217,346

Sonnenfels, Joseph von 63

Sophokles 554, 702

Sorkin, David 646

Sorokin, Pitrim A. 337

Soteriades, Georgios 263

Sowjetunion (UdSSR) 308, 322, 464, 470, 487 , $529,621,627$

Spanien 196, 457,673

Spann, Othmar 302

Sparta $495,55^{2}, 554,638,702$

Spengler, Oswald 264, 283, 287, 288-315, $317,332-334,343,346-349,35^{2}$ f., 369, $386,392,398,417,420,425,434-436$, $498,546,564,662,729-731$

Speyer $55^{1}$
Spiel, Hilde 459, 546

Spindler, Max 525-527,603

Spöckberger, Josef 55

Spörlein, Johann 172

Spranger, Eduard 236, 287, 306 f., 313, 326, 435

Sprengel, Peter 525, 710

Springer, Ferdinand 380

Springer, Julius 341,380

Spuler-Stegemann, Ursula 717

Stadelmeyer, Daniel Christian 113

Stadelmeyer, Rosine Luise, geb. Beck 113

Stählin, Friedrich 207

Stählin, Otto 231 f., 339

Stahl, Wilhelm 165

Stalin, Josef 308, 713, 715

Stalingrad 4,86

Stanford 596, 600

Staub, Hermann $366,368,376$

Stauffenberg, Alexander Graf Schenk von $497,507,637$

Steidle, Wolf 535

Stein, Benjamin 686

Stein, Charlotte von 245

Stein, J. A. 205

Stein-Hölkeskamp, Elke 708

Steinbach, Udo 589,714

Steinwenter, Artur 502

Stemberger, Günter 638f., 643, 716

Stengel, Paul 230

Stenglein, Melchior 366,376

Stern, Fritz 661, 694, 710, 719, 736

Stern, William $53^{\circ}$

Sternberger, Dolf 534

Stettin 292

Stettin, August Lebrecht 54

Steuben, Hans von 698

Stiehler, Wilhelm 4,13

Stier-Somlo, Fritz 259

Stifel, G. H. 186

Stinnes, Edmund 296

Stockholm 495

Stollberg-Rilinger, Barbara $706 \mathrm{f} ., 710$

Stolleis, Michael 396, 707

Stolz, Friedrich 211, 213, 229

Stone, Norman 617

Stotz, Peter 701

Stowe, Harriet Beecher 478

Strack, Hermann Leberecht 235, 334, 64, $\mathrm{f}$.

Strasburger, Hermann 502

Straßburg 134, 211, 213, 243, 274, 366

Straub, Johannes 492

Strauß, Franz Josef 699

Strecker, Karl 276

Strelin, Georg Gottfried 45f., 50

Strode, Hudson 400 
Stroheker, Karl Friedrich 496, 502, 506, 510 Struve, Gustav 158

Stuckart, Wilhelm 382, 392-394, 398, 546

Stürmer, Michael 598,618,622,625

Stufer, Adalbert 186

Sturz, Helfrich Peter 60

Stuttgart 54f., 64, 108, 128, 148, 158 f., 179, 201, 255 f., 298, 340, 471, 496, 513, 593, $620,643,646,702$

Suchenwirth, Richard 195-198

Südafrika 298, 384

Süddeutschland $38,43-45,53,55,59,63 \mathrm{f}$., $114,123,135,139,148,153,165,169-171$, $174,178,201,214,233,314$

Süßmilch, Johann Peter 84 .

Sueton, Gaius 535

Suez 457

Sulla Felix, Lucius Cornelius, Diktator 703

Sund, Georg 234f., 320, 358, 400, 408, 411, $4.15,423,4,27$ f., $432,445,447,454$ f., 485-488, 490, 493f., 497, 500-503, $505-507,510,5^{23}, 5^{26}, 537-540,543$, 545, 553, 557 f., 56o, 567 f., 571, 579, 699, 725

Swoboda, Heinrich $23^{2}$

Swoboda, Hermann 459

Sybel, Heinrich von 165, 190, 193-195

Syme, Ronald 509, 555

Syrien 234, 351, 586

Tagore, Rabindranath 586

Taizé ${ }_{4}, 66$

Tarn, William Woodthorpe 492

Tauberbischofsheim 211, 213

Tautenhayn, Josef 368

Teilhard de Chardin, Pierre 521 f., 538

Tel Aviv $645 \mathrm{f}$.

Tenfelde, Klaus 592

Terenz 251

Theodosius I., röm. Kaiser 643

Thesing, Curt 401

Theunissen, Michael 702

Thiele-Fredersdorf, Herbert 540

Thielemann, Christian 710

Thoma, Ludwig 457

Thomas, Louis 4,13

Thürauf, Ulrich 194 f., 198

Thüringen 274, 344

Thukydides 499, 555

Thumb, Albert 229

Thurn und Taxis, Karl Anselm von 54

Thurnau 14.4

Tigerstedt, Eugène N. 4.95

Tillich, Paul ${ }_{467}$

Tilly, Johann 't Serclaes von 24

Tirol 402, 4,05

Titel, Volker 127
Tizian 682

Tod, Markus N. $49^{2}$

Tönnesmann, Andreas 528

Torgau an der Elbe 462

Trabant, Jürgen 696

Träger, Claus 585

Trakl, Georg 688

Traube, Ludwig 222 f., 265,267

Traumann, Ernst 248

Traunstein 186

Treitschke, Heinrich von 162, 165, 171, 176, 186,192 f., 620

Trenck von Tonder, Moritz Flavius 100

Trenkle, Carl Hermann 186

Trient 634

Trier 645

Troeltsch, Ernst 529

Tröltsch, Georg Christian von 46, 68, 70-72, $94 \mathrm{f} ., 115 \mathrm{f}$.

Tröltsch, Johann Friedrich von 51, 68, 70, 95

Tröltsch, Walfried Daniel von 46, 86, 95

Trübner, Karl 258

Trüdinger, Hans 269, 331, $408 \mathrm{f}$.

Trunz, Erich $573 \mathrm{f}$.

Tschechien 473,668

Tschechoslowakei (ČSSR) 473f., $567,65^{2}$

Tschernjachowsk (Insterburg) 462, 470

Tübingen 20, 54, 75, 108, 161, 179, 186, 201 f., 244, 314, 323, 399, 423 f., 427, 431, $455,492,502,506,554,560,643,692$

Türkei 703,711

Tugendhat, Ernst 693

Turner, Henry A. 617

Turner, William 682

Ueding, Gert 716

Ulbricht, Walter 321, 475

Ulm 54, 100, 112, 158

Ullrich, Sebastian 664,707

Ullrich, Volker 620,653

Ungarn 121, 133, 448, 457

Unger, Friederike Helene $127 \mathrm{f}$.

Unger, Georg Friedrich 211 f., 228

Unger, Johann Friedrich 38

Unseld, Siegfried 531, 620,627, 657,670, $73^{2}$

Urban, Ralf 629

Urlichs, Ludwig von 211 f., 228

Usener, Hermann 214

Varzin 274

Veit, Moritz 181

Veith, Georg 234

Venedig 121

Verdun 277,326

Vereinigte Staaten von Amerika (USA) 121, $146,266,3_{16} 6,3_{18} 8,3^{20}, 3^{23}, 33^{0}, 3^{6} 5,457$, $47 \mathrm{o}, 47^{8}, 5^{19}, 544,57_{1}$ f., $582,59^{2}, 59^{6}$, 
598, 600 f., 606 f., 618, 63o, 647, 650-652, $654,680,716,718$

Verlaine, Paul 295

Versailles 173, 180, 268, 679

Vesper, Will 251 f., 275 f., 289 f., 330

Vidal-Naquet, Pierre $63^{2}$

Viel, Bernhard 710

Vietnam 461, 520

Vischer, Friedrich Theodor 2o, 169, 179-183

Vischer, Robert 180

Visp 63

Vittinghoff, Friedrich 502, 509

Völk, Joseph 170

Vogel, Hans-Jochen 518

Vogel, Johann Gottfried 70, 110

Vogt, Joseph 492, 5o6, 554, 63o, 634, 636

Voigt, Moritz 211, 213, 230

Voigtländer, Adam 28

Voigtländer, Esther Rosina s. Beck, Esther Rosina

Voigtländer, Gabriel 28

Voigtländer, Johann $28 \mathrm{f}$.

Volkelt, Hans 337, 385, 441, 530

Volkelt, Johannes 325 f., 329, 337

Volkert, Wilhelm $5 \mathbf{2 7}$

Volkmann, Hans 555

Volkmann, Hermann 592

Volkmann, Richard 211, 213, 229

Volkov, Shulamit $645 \mathrm{f}$.

Voltaire, François-Marie Arouet 63, 92, 346

Von der Leyen, Friedrich 403

Voß, Christian Friedrich 38,80

Voss, Johann Heinrich 562

Vossler, Karl 336, 398

Wachinger, Helga 688

Wachinger, Kristof 688

Wachter, Wilhelm 54,2

Wackernagel, Martin 326

Wade-Gery, Henry Theodore 499

Wagener, Hans-Jürgen 717

Wagenführ, Horst 302

Wagenknecht, Christian 562, $57^{2}$

Wagner, Richard 260, 368, 561, 710

Waibel, Aloys Adalbert (Theophilus Nelk) 119,201

Wais, Kurt 399

Wallerstein 134, 255

Wallis 63

Walser, Gerold 496 f., 502, 633

Walser, Martin 657, $73^{2}$

Waltershausen, Hermann Wolfgang von $532 \mathrm{f}$.

Wang, Samuel 691

Wapnewski, Peter $559,561 \mathrm{f}$.

Warnke, Martin 681

Warschau 322
Waser, Johann Heinrich 65

Washington 322

Washington, George $45^{1}$

Wassermann, Jakob 258

Watteau, Antoine 54,2

Weber, Alfred 534

Weber, Dietrich 458

Weber, Ekkehard 513

Weber, Hans von 272

Weber, Max 14, 32, 288, 504, 509, 529, 621, 630,723

Weber-Kellermann, Ingeborg 618

Wedemeyer, Maria von 697

Wegelin, Peter 697

Wegner, Christian $573 \mathrm{f}$.

Wegner, Ulrike 66o, 665

Wehler, Hans-Ulrich 464, 593,6o3, 6o5, 6o9, 613-628, 661, 714, 733, 735

Weigel, Adolf (Ernst Droem) 294f., 305

Weil, Rudolph 211

Weiler, Sophie Juliane 119

Weimann, Robert 584

Weimar 100, 193, 195, 237, 243, 247, 259, 272, 282 f., 288, 292, 295, 298-301, 304, 309, 316, $33^{2}$ f., 339-341, 343, 346, 349, 365, 401, 409, 433, 435, 581, 591, 651 f., 679,707 f., $710,728-730$

Weinrich, Harald $696 \mathrm{f}$.

Weis, Eberhard 526, 709

Weiss, Peter 477

Weiss, Yfaat 655

Weißenberger, Michael 702

Weizsäcker, Richard von $644,694,711$

Wekhrlin, Johann Marcel 95

Wekhrlin, Wilhelm Ludwig 42, 52, 54, 57, 59-69, 71-74, 86 f., 89-97, 102, 107, 109 f., 726

Welcker, Carl Theodor 164

Wellber, David E. 563

Welles, Charles Bradford 492

Wemding 565

Wende, Peter 705

Wendler, Johann 38, 52, 73

Wenger, Leopold $227 \mathrm{f}$.

Werner, Anton von 268

Wesel, Uwe 708

Wessel, Horst 4,12

Wessely, Karl 228

Westermayer, Adolf 205-207

Westermayer, Mathilde 207

Westfalen 465, 648, 695

Weygand, Johann Friedrich 38, 51, 53, 73, 109

Wichern, Johann Heinrich 14,1, 144

Wickert, Lothar 492-494

Widenmann, Gustav 16o, 177 
Widmann, Adolf 177

Widor, Charles-Marie 314,

Wieacker, Franz 698

Wieckenberg, Ernst-Peter 510-514, 526, 564, 566-569, 573f., 58o-586, 588 f., 591-593, 597 f., 6oo-6o6, 610-62o, 623-627, $629-63^{2}, 634-639,641,643-645$, 648-652, 654f., 657, 661 f., 699, 705, 712 , $714,725,73^{2}-735$

Wiegand, Theodor 231

Wiegler, Paul 348

Wieland, Christoph Martin 53, 113, 205, 294, 562

Wiemann, Mathias $5^{18}$

Wiemer, Hans-Ulrich 703

Wiemer, Horst 411, 413, 415, 435, 445-448, $45^{1-453}, 455^{-460}, 465,470-479,5^{21}, 5^{27}$, $540-543,545,560,571$

Wien 24, 52, 59-64, 89, 98, 113, 115, 128, 195, 262, 289 f., 345, 352 f., 355 f., 358, 419,

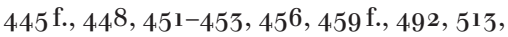
$528,559,643,681 \mathrm{f}$.

Wiesbaden 497,668

Wiese, Benno von $558 \mathrm{f}$.

Wilamowitz-Moellendorff, Ulrich von 214 , $216,221 \mathrm{f}, 239,335 \mathrm{f}$.

Wilckens, Ulrich 233

Wilbrandt, Adolf $181 \mathrm{f}$.

Wildung, Dietrich 681

Wilhelm I., Deutscher Kaiser 171, 180, 183, $268 \mathrm{f}$.

Wilhelm II., Deutscher Kaiser 219, 247 f., 253, 274, $276 \mathrm{f}$., 343, 405, 408, 592, 6o6, 709

Wilhelm, Adolf 492

Wilke, Gustav 395

Wilkinson, Lancelot Patrick 634

Willems, Paul $476 \mathrm{f}$.

Willms, Johannes 709

Willoweit, Dietmar 382, 394

Wilpert, Gero von 717

Windelband, Wilhelm 211, 213 f., 230

Winckelmann, Johann Joachim 347,562

Winkler, Heinrich-August 623, 707

Winter, Carl 296, 34,1 f.

Winter, Ernst Karl 336

Winterling, Aloys 703

Winterthur 154

Wissowa, Georg 230, 232, 267

Witkowski, Georg $\mathbf{3 2 5}$

Witsch, Joseph Caspar 480
Wittenberg 37,85

Wittenburg, Andreas $63^{2}$

Wittig, Friedrich 548

Wittmann, Reinhard 14, 109

Wöhrle, Oskar 405

Wölfflin, Eduard 209

Woinovich, Peter von 459

Wolf, Hubert 695, 709

Wolfenbüttel 710

Wolfram, Herwig 715

Wolfram von Eschenbach 252, 561

Wolfskehl, Karl 289 f., 294, 304, 315, 338, 342,433

Wolters, Reinhard 702

Worms 247

Woytt, Gustave 323

Woytt-Secretan, Marie 320, 323

Wucherer, Johann Friedrich 14,-14,8

Wucherer, Johann Philipp ${ }_{4} 6$

WuDunn, Sheryl 715

Württemberg 42, 45, 47, 50, 52, 59, 64, f., 7o, 74 f., $81,86,102,110,113,115,118,158$, $178 \mathrm{f} ., 292$

Würzburg 185f., 211 f., 214, 226, 298, 500, $5^{02}, 5^{18}, 535,574,659,696$

Wüst, Fritz R. 492

Wüst, Walther 4,85

Wundt, Wilhelm 325

Wurche, Ernst 277, 284

Zamoyski, Adam 667

Zanker, Paul 683,698, 705

Zapf, Georg Wilhelm 67, 111

Zborowski, Mark 647

Zedler, Johann Heinrich 40

Zeile, Christine 618, 647,664

Zeilinger, Anton 690

Zeller, Eduard 214

Ziegenschacht 28

Ziegler, Hendrik 681

Ziegler, Theobald 24,4

Zielinski, Thaddäus 228

Zimmermann, Bernhard 699, $701 \mathrm{f}$.

Zinknagel, Karl Friedrich Bernhard $50 \mathrm{f}$.

Zöckler, Otto 209, 235

Zorn, Jakob 122

Zürich 65, 153f., 156, 16o, 177, 189, 354,f., 36of., 573, 604f., 6o7, 66o, 701, 703, 706

Zunz, Leopold $65^{\circ}$

Zweig, Stefan 318, 365, 518 